\title{
Menstruations douloureuses chez les adolescentes
}

\author{
Olga Kciuk MD MSc, Sari Kives MD
}

Citation : CMAJ 2021 April 19;193:E560. doi : 10.1503/cmaj.201972-f

Voir la version anglaise de l'article ici : www.cmaj.ca/lookup/doi/10.1503/cmaj.201972

\section{1}

La dysménorrhée - menstruations pénibles et

douloureuses - touche 50\%-90\% de la population adolescente menstruée et constitue la principale cause d'absentéisme scolaire récurrent dans ce groupe

Un tiers des adolescentes menstruées s'absentent de l'école ou évitent les activités sportives en raison de la dysménorrhée ${ }^{1}$. Pour réduire autant que possible les répercussions sur le développement scolaire et social, les médecins doivent conseiller de manière proactive les patientes sur les options de traitement des douleurs menstruelles ${ }^{1-3}$. Le traitement de première intention peut être commencé avant qu'un diagnostic précis soit posé2.

Jusqu'à $90 \%$ des cas de dysménorrhée chez les adolescentes ne sont pas liés à une pathologie pelvienne (dysménorrhée primaire) et sont dus à une production excessive de prostaglandines La présence de signes cliniques évocateurs d'une dysménorrhée secondaire (encadré 1) doit inciter à réaliser une échographie pelvienne et peut justifier une consultation chez un gynécologue $e^{2,3}$.

Encadré 1 : Signes cliniques évocateurs d'une dysménorrhée secondaire ${ }^{2,3}$

- Apparition des symptômes immédiatement après le début des premières règles

- Dysménorrhée à aggravation progressive

- Saignements anormaux (notamment irréguliers) et douleur

- Antécédents familiaux ou personnels d'anomalies rénales ou d'anomalies congénitales (notamment rachidiennes, cardiaques ou gastro-intestinales)

- Douleur en milieu de cycle ou acyclique

- Dyspareunie

- Antécédents familiaux d'endométriose
L'administration prophylactique à court terme d'anti-inflammatoires non stéroïdiens (AINS) est le traitement de première intention

Le naproxène, l'ibuprofène et les autres AINS sont d'une efficacité équivalente (nombre de sujets à traiter [NST] de 3 pour le soulagement de la douleur chez les personnes atteintes de dysménorrhée primaire) ${ }^{4}$. La personne doit prendre des doses entières avec des aliments et à intervalles réguliers; aucune dose ne doit être sautée. L'administration doit commencer 1 ou 2 jours avant le début des menstruations (si elles sont prévisibles) ou au premier signe de saignement ou de douleur, et doit se poursuivre pendant les 2 ou 3 jours suivant le début des saignements ${ }^{2,3}$.

Des médicaments hormonaux combinés ou uniquement à base de progestine peuvent être ajoutés comme traitement d'appoint

Le NST des contraceptifs oraux combinés pour obtenir un soulagement de la dysménorrhée primaire est de $5^{5}$. Chez les adolescentes, il convient de choisir des contraceptifs oraux combinés dont les doses d'éthinylestradiol sont supérieures à $30 \mu \mathrm{g}$ pour favoriser le maintien de la santé osseuse ${ }^{6}$. L'administration continue procure un soulagement plus efficace que l'administration cyclique habituelle ${ }^{2,3}$. Les systèmes intra-utérins contenant du lévonorgestrel et l'implant à l'étonogestrel sont également des traitements de première ligne sûrs et efficaces ${ }^{2,3,7}$.

L'endométriose est la cause la plus fréquente de dysménorrhée secondaire chez les adolescentes Jusqu'à $70 \%$ des adolescentes qui subissent une laparoscopie pour une dysménorrhée réfractaire au traitement par AINS et à l'hormonothérapie présentent une endométriose $\mathrm{e}^{3}$. Si la dysménorrhée persiste au-delà de 3 mois malgré un traitement de première intention adéquat, il est justifié d'orienter la patiente vers un gynécologue. 


\section{Références}

1. Armour M, Ferfolja T, Curry C, et al. The prevalence and educational impact of pelvic and menstrual pain in Australia: a national online survey of 4202 young women aged 13-25 years. J Pediatr Adolesc Gynecol 2020;33:511-8.

2. Burnett M, Lemyre M. No. 345-primary dysmenorrhea consensus guideline. J Obstet Gynaecol Can 2017;39:585-95.

3. American College of Obstetricians and Gynecologists. Dysmenorrhea and endometriosis in the adolescent. ACOG Committee opinion no. 760 . Obstet Gynecol 2018;132:e249-58.

4. Marjoribanks J, Ayeleke RO, Farquhar C, et al. Nonsteroidal anti-inflammatory drugs for dysmenorrhoea. Cochrane Database Syst Rev 2015;(7):CD001751.

5. Wong $\mathrm{CL}$, Farquhar $\mathrm{C}$, Roberts $\mathrm{H}$, et al. Oral contraceptive pill for primary dysmenorrhea. Cochrane Database Syst Rev 2009;(4):CD002120.

6. Golden NH. Bones and birth control in adolescent girls. J Pediatr Adolesc Gynecol 2020;33:249-54.

7. Bayer LL, Hillard PJA. Use of levonorgestrel intrauterine system for medical indications in adolescents. J Adolesc Health 2013;52:S54-8.

Intérêts concurrents : Aucun déclaré.

Cet article a été révisé par des pairs.

Affiliations : Département d'obstétrique et de gynécologie, Université de Toronto (Kciuk, Kives); Hôpital SickKids, Toronto (Kives), Toronto, Ont.

Propriété intellectuelle du contenu : Il s'agit d'un article en libre accès distribué conformément aux modalités de la licence Creative Commons Attribution (CC BY-NC-ND 4.0), qui permet l'utilisation, la diffusion et la reproduction dans tout médium à la condition que la publication originale soit adéquatement citée, que l'utilisation se fasse à des fins non commerciales (c.-à-d., recherche ou éducation) et qu'aucune modification ni adaptation n'y soit apportée. Voir : https://creativecommons.org/ licenses/by-nc-nd/4.0/deed.fr.

Correspondance : Olga Kciuk, olga.kciuk@mail.utoronto.ca 